\title{
Role of Order in the Mechanism of Charge Transport across Single- Stranded and Double-Stranded DNA Monolayers in Tunnel Junctions
}

Nipun Kumar Gupta, ${ }^{\text {II }}$ Edward A. Wilkinson, ${ }^{\text {II }}$ Senthil Kumar Karuppannan, Lily Bailey, Ayelet Vilan, * Ziyu Zhang, Dong-Chen Qi, Anton Tadich, Eimer M. Tuite,* Andrew R. Pike,* James H. R. Tucker,* and Christian A. Nijhuis*

Cite This: J. Am. Chem. Soc. 2021, 143, 20309-20319

Read Online

ACCESS 1

Llll Metrics \& More

Article Recommendations

Supporting Information

ABSTRACT: Deoxyribonucleic acid (DNA) has been hypothesized to act as a molecular wire due to the presence of an extended $\pi$-stack between base pairs, but the factors that are detrimental in the mechanism of charge transport (CT) across tunnel junctions with DNA are still unclear. Here we systematically investigate CT across dense DNA monolayers in large-area biomolecular tunnel junctions to determine when intrachain or interchain CT dominates and under which conditions the mechanism of CT becomes thermally activated. In our junctions, double-stranded DNA (dsDNA) is 30 -fold more conductive than single-stranded DNA (ssDNA). The main reason for this large change in

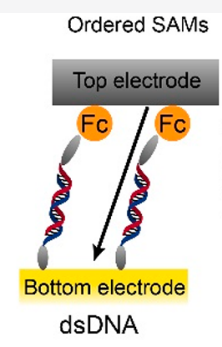

Coherent Tunneling

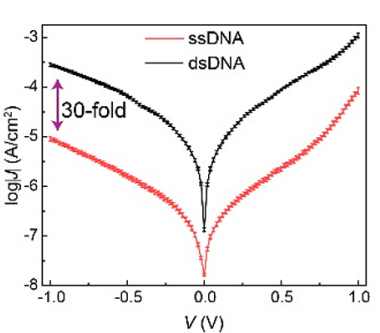

ssDN

Intrachain Hopping Interchain Hopping conductivity is that dsDNA forms ordered monolayers where intrachain tunneling dominates, resulting in high CT rates. By varying the temperature $T$ and the length of the DNA fragments in the junctions, which determines the tunneling distance, we reveal a complex interplay between $T$, the length of DNA, and structural order on the mechanism of charge transport. Both the increase in the tunneling distance and the decrease in structural order result in a change in the mechanism of CT from coherent tunneling to incoherent tunneling (hopping). Our results highlight the importance of the interplay between structural order, tunneling distance, and temperature on the CT mechanism across DNA in molecular junctions.

\section{INTRODUCTION}

Deoxyribonucleic acid (DNA) has been hypothesized to act like a molecular wire due to the presence of a $\pi$-stack ${ }^{1,2}$ and, therefore, can have potential applications in DNA-based computing $^{3-6}$ and switches. ${ }^{7,8}$ To realize these goals, it is important to establish the mechanisms of charge transport (CT) across DNA in solid-state devices, but such studies are challenging and have in the past resulted in controversial results. ${ }^{1}$ A key reason for the controversy is that many of the studies have assumed that the DNA molecules exist in a stretched conformation inside tunnel junctions, but, in reality, DNA is highly flexible in nature and, therefore, likely forms disordered structures. In addition, most studies have been based on rather invasive single-molecule techniques which suffer from poor reproducibility, where it is challenging to ascertain the conformation of the DNA or how the DNA molecules interact or connect with the electrodes. This paper describes the mechanism of charge transport across disordered monolayers of single-stranded DNA (ssDNA) and ordered monolayers of double-stranded DNA (dsDNA) supported by ultraflat $\mathrm{Au}$ electrodes in contact with large-area noninvasive
EGaIn electrodes (see Figure 1, EGaIn is an eutectic alloy of $\mathrm{Ga}$ and $\mathrm{In})$ as a function of the number of base pairs $(n=15$, $20,25$, and 30$)$ and temperature $(T=150-330 \mathrm{~K})$. The advantage of this approach is that the DNA molecules are preorganized in monolayers whose structure can be verified using independent techniques prior to the fabrication of the well-known, noninvasive EGaIn top contact. ${ }^{9,10}$ The tunneling rates across junctions with dsDNA are 30 times higher than those junctions with ssDNA. This difference in conductivity is mainly due to the formation of highly ordered dsDNA selfassembled monolayers (SAMs) along which charges can efficiently tunnel coherently. This is in stark contrast to disordered ssDNA SAMs where charges have to tunnel incoherently between and along DNA chains. Our results

Received: September 9, 2021

Published: November 26, 2021 
A

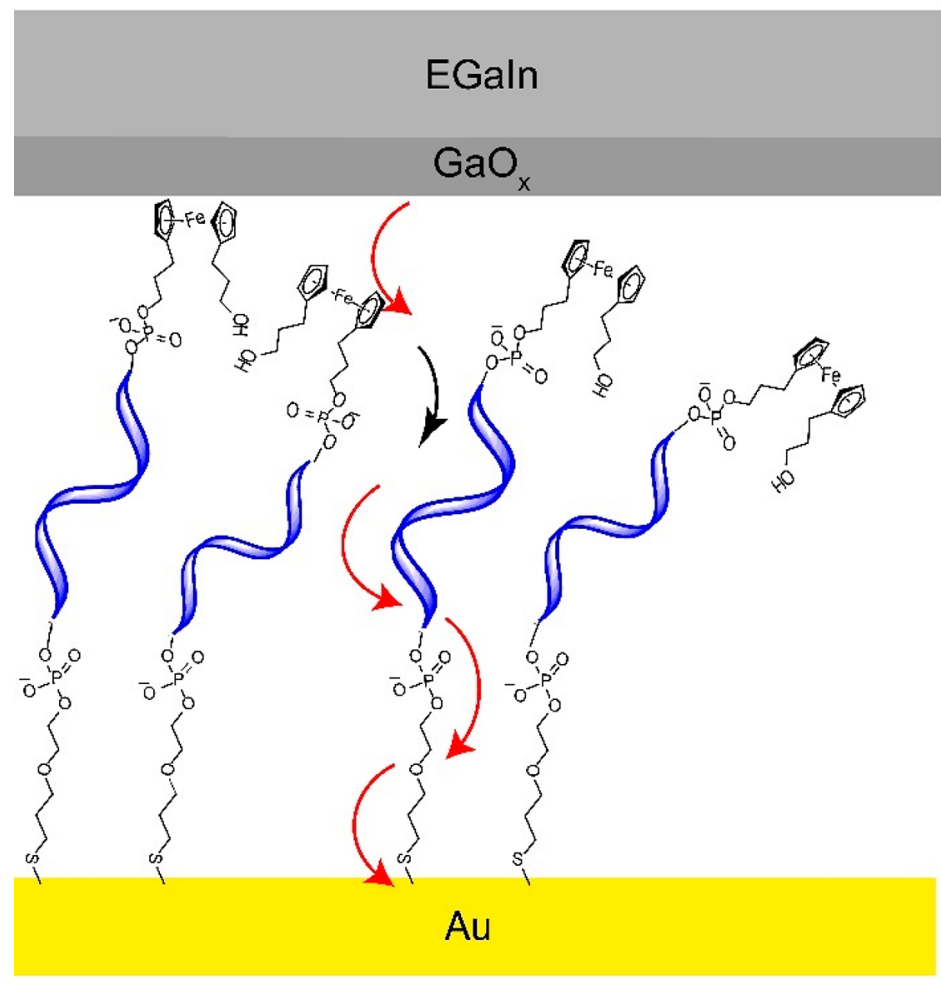

B

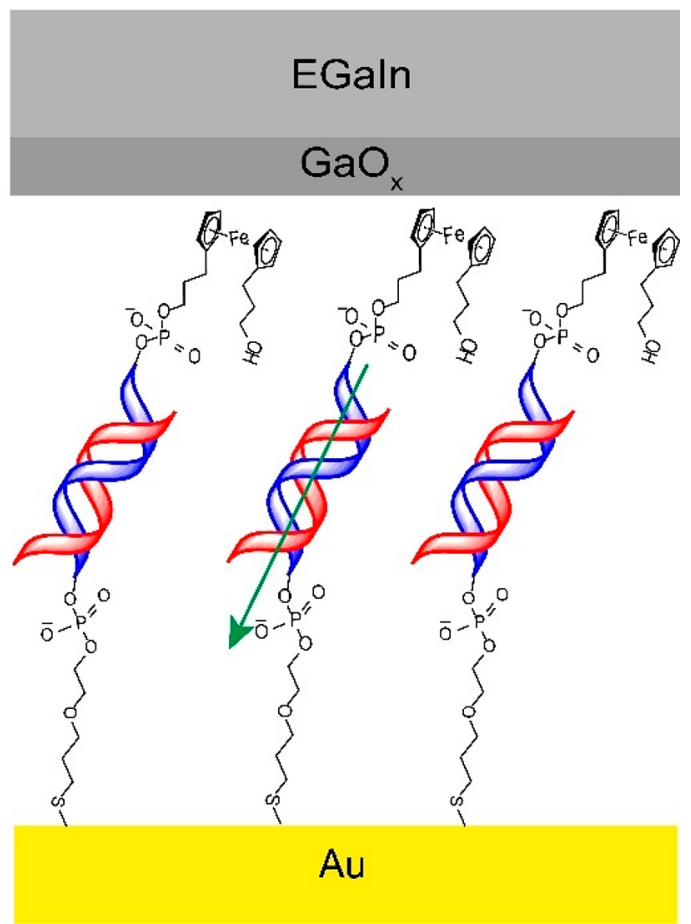

Figure 1. (A) Schematic representation of the Au-linker-ssDNA $15-\mathrm{Fc} / / \mathrm{GaO}_{x} / \mathrm{EGaIn}$ junction where incoherent tunneling dominates $\mathrm{CT}$ : intra (red) and inter (black) hopping is indicated by the curved arrows. (B) Schematic representation of the Au-linker-dsDNA 15 -Fc//GaOx/EGaIn junction, where the mechanism of CT is coherent tunneling represented by a green arrow. Here, "//" represents a van der Waals contact, "/" represents the contact between the $\mathrm{GaO}_{x}$ and EGaIn, and "-" represents a chemical contact.

help to improve our understanding of CT rates across DNA fragments and especially how (dis)order affects the observed tunneling efficiencies.

The various mechanisms of CT across DNA can be broadly divided into coherent tunneling (which is essentially independent of temperature) and incoherent tunneling (which is thermally activated and is also called hopping). The measured current density $\left(J\right.$, in $\left.\mathrm{A} / \mathrm{cm}^{2}\right)$ in the coherent tunneling regime for tunneling through a barrier of width $d$ (in $\mathrm{nm}$ ) is described by the general tunneling equation (eq 1)

$$
J=J_{0} \exp (-\beta d)
$$

where $J_{0}$ is the pre-exponential factor and $\beta$ is the tunneling decay coefficient (in $\mathrm{nm}^{-1}$ ). CT in the hopping regime for overbarrier transport with activation energy $\left(E_{\mathrm{a}}\right.$, in $\left.\mathrm{eV}\right)$ is described by the Arrhenius equation (eq 2)

$$
J=A \exp \left(-\frac{E_{\mathrm{a}}}{k_{\mathrm{B}} T}\right)
$$

where $k_{\mathrm{B}}$ is the Boltzmann constant, $A$ is the pre-exponential factor, and $T$ is the absolute temperature (in $\mathrm{K}$ ). The measured current is likely the summation of several contributions involving both activationless and thermally activated components depending on $d$ and $T$, where the observed $J\left(J_{\text {obs }}\right)^{11,12}$ is given by eq 3

$$
J_{\text {obs }}=J_{0} \exp (-\beta d)+A \exp \left(-\frac{E_{\mathrm{a}}}{k_{\mathrm{B}} T}\right)
$$

At sufficiently high $T$ and large $d$, the second term likely dominates, but at low $T$ and small values of $d$ the first term is likely to dominate. In this work, we show that structural order is also important to consider where the first term dominates CT for ordered structures but the second term dominates CT for tunneling across disordered structures at a given $T$ and $d$.

CT has been studied across various secondary structures of DNA, including the canonical B-form and G-quadruplex, ${ }^{13}$ as well as its analogues, such as peptide nucleic acid (PNA) ${ }^{13}$ in solution and in solid-state tunnel junctions. Most solution-state studies suggest that short DNA and PNA strands (both ss and ds) undergo charge transfer with a high $\beta$, suggesting a superexchange mechanism $\left(\beta=0.6-1.0 \AA^{-1}\right)$ that dominates charge transfer. ${ }^{14-16}$ In contrast, long dsDNA strands undergo charge transfer with a low $\beta\left(\beta \sim 0.1 \AA^{-1}\right)$, which suggests that charge transfer is dominated by hopping. ${ }^{14,15,17-19}$ Recent CT studies have shown that CT across SAMs of G-quadruplex DNA proceeds by hopping for $d=20-70 \mathrm{~nm} .{ }^{20}$ Interestingly, Xiang et al. ${ }^{21}$ reported that resistance increases linearly with the length as expected for incoherent tunneling, but they also observed oscillations in the resistance for a dsDNA stack with alternating segments of $G$ and $C$. This implies that CT proceeds at least partially coherently across the base pairs along the length of the DNA strand in single-molecule break junctions with stacked G-C base pairs. A periodic oscillation caused by the number of $G$ units superimposed on the linear length dependence was observed, which was attributed to a combination of coherent and incoherent tunneling across the dsDNA junctions. Venkatramani et al. ${ }^{18}$ also observed a combination of coherent and incoherent tunneling in dsPNA 
in solution with electrochemical junctions. The charge transfer rates measured in their experiments cannot be explained by a coherent superexchange (a form of long-range coherent tunneling) or hopping mechanism alone, with simulations suggesting a combination of coherent superexchange and hopping models in the near-resonant limit. These studies, however, lack temperature-dependent CT characterization, which is needed to discriminate between coherent (eq 1) and incoherent tunneling (eq 2).

The redox potentials of the individual nucleotides usually fall outside the electrochemical window. Therefore, often solutionstate charge transfer across DNA is studied by tethering a redox unit, such as ferrocene $(\mathrm{Fc}),{ }^{22-24}$ methylene blue, ${ }^{25,26}$ Nile Blue, ${ }^{27}$ or daunomycin, ${ }^{28}$ to the termini of DNA strands to facilitate charge injection. These studies have demonstrated that charge transfer occurs through the $\pi$-stack and not the sugar-phosphate backbone, ${ }^{28,29}$ highlighted the deleterious effect of a base-pair mismatch in charge transfer rates across DNA due to perturbation of the $\pi$-stack, ${ }^{27,29}$ and explored charge transfer in lengths of up to $34 \mathrm{~nm}$ in DNA wires. ${ }^{27}$ In addition, the characterization of DNA monolayers is facilitated by the presence of a redox-active tagas, for instance, it can be detected electrochemically from which the surface coverage $(\Gamma$, in $\mathrm{mol} / \mathrm{cm}^{2}$ ) can be quantified.

Studies of CT across DNA in tunnel junctions have attempted to resolve the question of whether dsDNA or ssDNA has a larger electrical conductivity. In principle, the $\pi$ stack of dsDNA can provide a conduction channel that is absent in ssDNA and hence dsDNA should be more conductive than ssDNA. ${ }^{1}$ This assumption has been confirmed in several studies, ${ }^{21,30-34}$ but an exception exists where the opposite conclusion was reached based on findings obtained from only one junction. ${ }^{35}$ In support of dsDNA being more conducting, perturbation of the $\pi$-stack has a deleterious effect on the CT rates in dsDNA tunnel junctions. ${ }^{35,36}$ However, systematic studies as a function of the number of base pairs (or d) or temperature are lacking, and the role of structure order has not been identified. For example, most studies involving charge transfer rates (determined electrochemically) use surface coverages of DNA SAMs in the range of $10^{-12}$ $-10^{-11} \mathrm{~mol} / \mathrm{cm}^{2}, 18,22,24,26,28,37$ which is well below the theoretical maximum surface coverage of $\sim 3.4 \times 10^{-10} \mathrm{~mol} /$ $\mathrm{cm}^{2}{ }^{38}$ Many of these monolayers are likely composed of disordered regions with the molecules in the lying-down phase. $^{39,40}$ The conformation of DNA in single-molecule tunnel junctions, and how it interacts with the electrodes is usually unknown. This lack of control along with a lack of systematic studies as a function of the number of base pairs and temperature, complicate the interpretation of results obtained from molecular tunnel junctions with DNA. ${ }^{21,30,41}$ An exception to the limitations of these investigations are the studies by Porath and co-workers who were able to image single DNA strands followed by $J(\mathrm{~V})$ and $J(V, T)$ measurements using a conductive probe technique (but these studies rely on a low number of junctions due to the complexity of their experiments). ${ }^{20,42}$

Herein, we found that dsDNA is at least 30 -fold more conductive than ssDNA in tunnel junctions based on SAMs of DNA supported by Au electrodes and in contact with EGaIn top electrodes. The DNA strands were functionalized with Fc at one terminus to facilitate charge injection and with a thiol group at the other to facilitate SAM formation on $\mathrm{Au}$ electrodes. The SAMs of dsDNA are well-packed and show a transition from activationless coherent tunneling to thermally activated incoherent tunneling as a function of $d$ (by changing the number of base-pairs) due to efficient CT involving tunneling along the $\pi$-stack. In stark contrast, ssDNA forms disordered SAMs and CT along ssDNA proceeds by thermally activated tunneling for all investigated $d$, indicating that interchain hopping limits the observed CT rates. Our study illustrates the importance of structural order in (bio)molecular wires and helps to resolve, at least partially, the discrepancies in earlier studies.

\section{RESULTS AND DISCUSSION}

Description of the Junctions. Figure 1 shows a schematic representation of the Au-linker-DNA- $\mathrm{E}, \mathrm{c} / / \mathrm{GaO}_{x} / \mathrm{EGaIn}$ junctions, where the DNA is attached at its 3 ' end to the Au surface via a metal thiolate bond (the $-\mathrm{S}-\left(\mathrm{CH}_{2}\right)_{3}-\mathrm{O}-\left(\mathrm{CH}_{2}\right)_{2}-$ $\mathrm{PO}_{4}^{-}-$linker) and at its $5^{\prime}$ end to the $\mathrm{Fc}$ tag via a $-\left(\mathrm{CH}_{2}\right)_{3}-$ $\mathrm{PO}_{4}^{-}-$linker. The EGaIn represents the top electrode (EGaIn = eutectic alloy of Ga and In, 3:1 ratio by weight), and the bottom electrode is an ultraflat template stripped $\mathrm{Au}$ surface (the experimental methods are described in the Supporting Information). We synthesized the ferrocene-modified ssDNA and their complementary strands as per reported procedures using phosphoramidite chemistry (see sections S1 and S2). ${ }^{43,44}$ To study CT across the DNA monolayers as a function of $d$, we prepared DNA with four different sequence lengths as indicated by the total base number, $n=15,20,25$, and 30 , where the 30 -mer DNA is two repeats of the 15-mer. Although we considered that there would be no issue in investigating any DNA sequence using this technique, in choosing these particular DNA base sequences (presented in Table S2), we ensured that (i) there would be limited scope for intramolecular folding via base pairing (which might otherwise be an issue for junctions with ssDNA), (ii) the smallest 15-mer sequence (which is then extended in steps of 5 bases up to a complete repeat in the 30-mer) would contain roughly equal amounts of purines and pyrimidines, and (iii) no one base would be the same beyond a run of two bases in any of the strands investigated. Figure 1 shows schematically that the

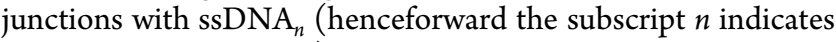
the number of bases) suffer from disorder while those junctions with $\mathrm{dsDNA}_{n}$ are well-ordered; this difference in order has a substantial influence on the mechanism of CT as discussed in detail below.

DNA Monolayer Characterization with Cyclic Voltammetry (CV). Solutions of ssDNA and their complementary strands were prepared by dissolving the purified DNA in aqueous buffers (see section S3 for details). We used templatestripped Au substrates to form the SAMs as template-stripping produces smooth bottom electrodes with minimal defects; these surfaces result in tunnel junctions with low leakage currents (induced by defects) and higher reproducibility as compared to as-deposited metal electrodes (see section S3.3 for details). ${ }^{45,46}$ SAMs of ssDNA on Au surfaces by incubation of $\mathrm{Au}$ substrates in aqueous buffered ssDNA solutions for $2 \mathrm{~h}$ under ambient conditions. Each ssDNA SAM on Au was immersed in an aqueous buffered solution with its complementary strand at $70{ }^{\circ} \mathrm{C}$ for $10 \mathrm{~min}$ to obtain the corresponding dsDNA SAM on Au (see section S3.4 for details).

We characterized the SAMs with $\mathrm{CV}$ as the Fc tags are redox-active, thus allowing us to quantify the surface coverage of the $\mathrm{Fc}$ units ( $\Gamma$ in $\mathrm{mol} / \mathrm{cm}^{2}$; see section $\mathrm{S} 3.5$ for 
(A)

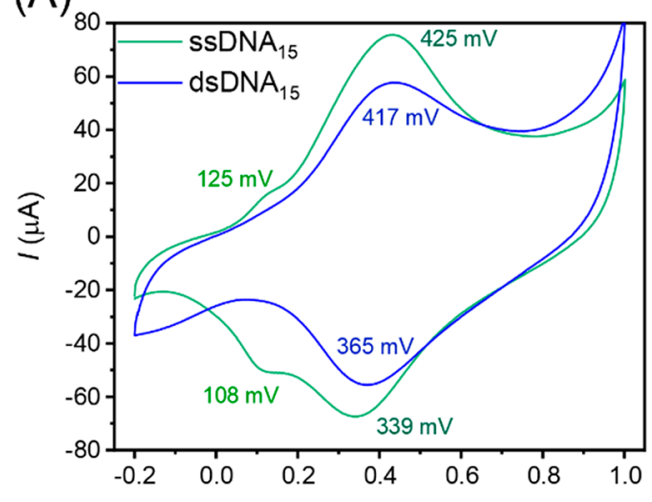

$(\mathrm{C})_{80}$

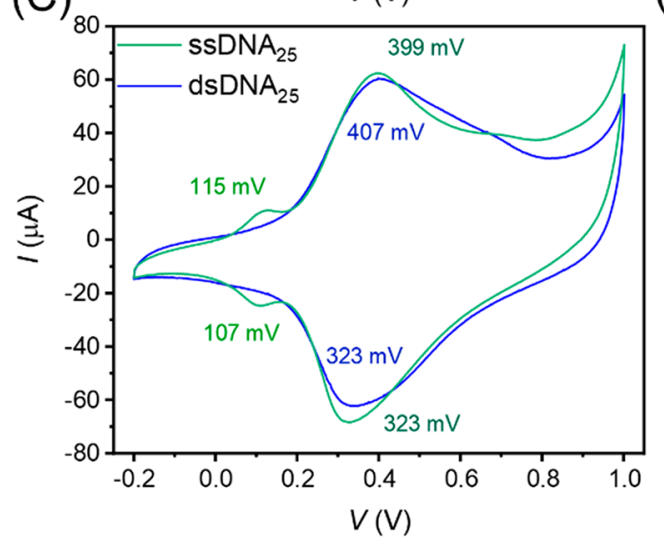

(B)

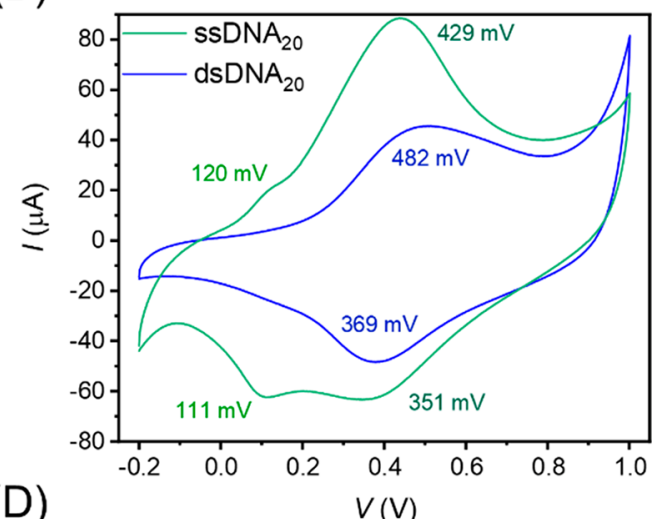

(D)

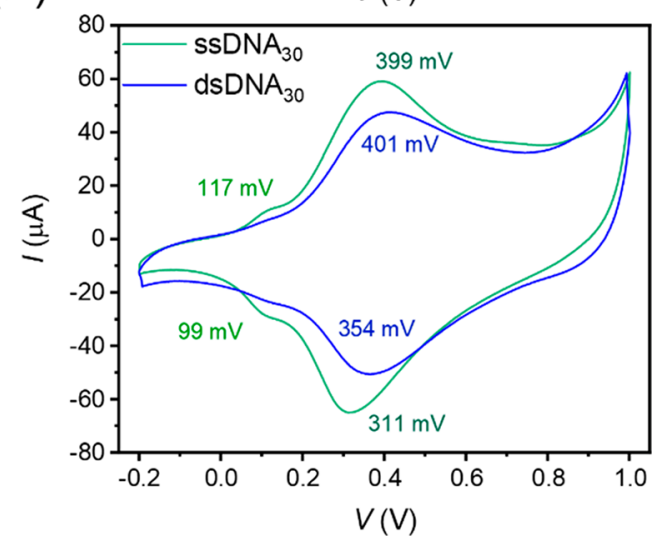

Figure 2. Representative cyclic voltammograms for SAMs of Fc terminated ssDNA $n$ (green) and dsDNA $n$ (blue) $(n=($ A) 15 , (B) 20, (C) 25 , and (D) 30) on Au, recorded against a $\mathrm{Ag} / \mathrm{AgCl}$ reference electrode with aqueous $1.0 \mathrm{M} \mathrm{NaClO}_{4}$ and $10 \mathrm{mM} \mathrm{NaH}_{2} \mathrm{PO}_{4}$ at $\mathrm{pH} 8.0$ as the electrolyte at a scan rate of $1.0 \mathrm{~V} / \mathrm{s}$. The peak oxidation and reduction potentials are denoted in the respective colors.

experimental details). In addition, the $\mathrm{CV}$ data can resolve whether the Fc units are primarily located at top of the SAMs (as expected for ordered SAMs) or are buried within the SAM (as expected for disordered SAMs) since the latter may result in a prepeak. ${ }^{47-50}$ Figure 2 shows representative cyclic voltammograms for monolayers of $\mathrm{ssNA}_{n}$ and $\mathrm{dsDNA}_{n}$ on Au with $n=15,20,25$, and 30, and Table S3 summarizes the electrochemical parameters over three separate experiments. Figure $2 \mathrm{~A}$ shows that the $\mathrm{CV}$ of $\operatorname{ssDNA}_{15} \mathrm{SAMs}$ consists of two peaks with the first peak oxidation potential at $E_{\mathrm{pa}, \mathrm{I}}=125 \mathrm{mV}$. This peak indicates regions in the monolayers where the $\mathrm{Fc}$ units are bent back into the SAM (back bending) and may be in close contact with the $\mathrm{Au}$ electrode, or it indicates disordered regions with the molecules in the flat-lying phase. $^{47,50}$ Given the large $\Gamma$ of both types of SAMs (see below), we believe that the prepeak is caused by back bending. The second peak dominates the $\mathrm{CV}$ at $E_{\mathrm{pa}, \mathrm{II}}=425 \mathrm{mV}$ which is close to the oxidation potential typically encountered for Fc. $^{47,50}$ In contrast, for SAMs of dsDNA ${ }_{15}$, we observe a single oxidation peak at $E_{\mathrm{pa}}=417 \mathrm{mV}$. These observations suggest that a considerable amount of a disordered phase is present in the ssDNA 15 SAM, and, thus, that the hybridization reduces the disorder considerably in the dsDNA. We obtained $\Gamma$ by integration of the peak area which reveals that $\mathrm{dsDNA}_{15}(2.23$ $\left.\pm 0.32 \times 10^{-10} \mathrm{~mol} / \mathrm{cm}^{2}\right)$ has a $40 \%$ lower $\Gamma$ than that of the $\operatorname{ssDNA}_{15} \operatorname{SAM}\left(3.71 \pm 0.17 \times 10^{-10} \mathrm{~mol} / \mathrm{cm}^{2}\right)$. The observed lower $\Gamma$ of dsDNA is consistent with our expectations as dsDNA has a larger surface volume as compared to ssDNA. ${ }^{22,51-53}$ The $\Gamma$ values for ssDNA SAMs are comparable to those of Fc alkanethiolates, ${ }^{54}$ and the estimated maximum $\Gamma$ for ssDNA is similar to the theoretically calculated maximum $\Gamma$ of $\sim 3.4 \times 10^{-10} \mathrm{~mol} / \mathrm{cm}^{2}{ }^{38}$ The DNA SAMs with $n=20,25$, and 30 , behave similarly. Therefore, we conclude that we formed one of the densest SAMs of DNA reported in the literature.

DNA Monolayer Characterization with Photoelectron Spectroscopy. We further characterized the ssDNA and dsDNA SAMs with angle-resolved X-ray photoelectron spectroscopy (AR-XPS) to establish the quality of the SAMs from the $S 2 p$ spectra (the experimental details are given in section S3.6). For the sake of completion, Figures S13-S17 show the Au $4 f, \mathrm{C} 1 s, \mathrm{O} 1 s, \mathrm{~N} 1 s, \mathrm{P} 2 p$ and S $2 p$ spectra, at normal emission (NE) and grazing emission (GE), and the peak assignments were taken from Vilar et al. ${ }^{55}$ In general, in $S$ $2 p$ spectra recorded from thiolate SAMs, often two peaks can be observed with peak $S_{1}$ corresponding to the chemisorbed $\mathrm{S}-\mathrm{Au}$ bond at $\sim 162.0 \mathrm{eV}$ and peak $\mathrm{S}_{2}$ corresponding to physisorbed $\mathrm{S}$ at $\sim 163.2 \mathrm{eV}^{45}$ Figure $\mathrm{S} 18 \mathrm{~A}$ shows the $\mathrm{S} 2 p$ spectra recorded at $\mathrm{NE}$ and $\mathrm{GE}$ for $\mathrm{ssDNA}_{15}$ which are dominated by the $S_{1}$ peak along with a small contribution from $S_{2}$. Peak $S_{2}$ is more intense at GE than at NE, which suggests that most of the physisorbed sulfur is present on the SAM. Similar observations were made for all the other ssDNA and dsDNA SAMs. We note that no S $2 p$ signal was detected for dsDNA $_{30}$ at GE which we attribute to strong signal attenuation by the thick layer of dsDNA. These XPS results confirm that the DNA monolayer is anchored to the Au surface via $\mathrm{Au}-\mathrm{S}$ bonds.

DNA SAM Thickness Determination. We estimated the thickness of the ssDNA and dsDNA SAMs with ellipsometry 
(see section S3.7 for experimental details). Figure 3 shows the measured thickness for ssDNA and dsDNA SAMs as a function

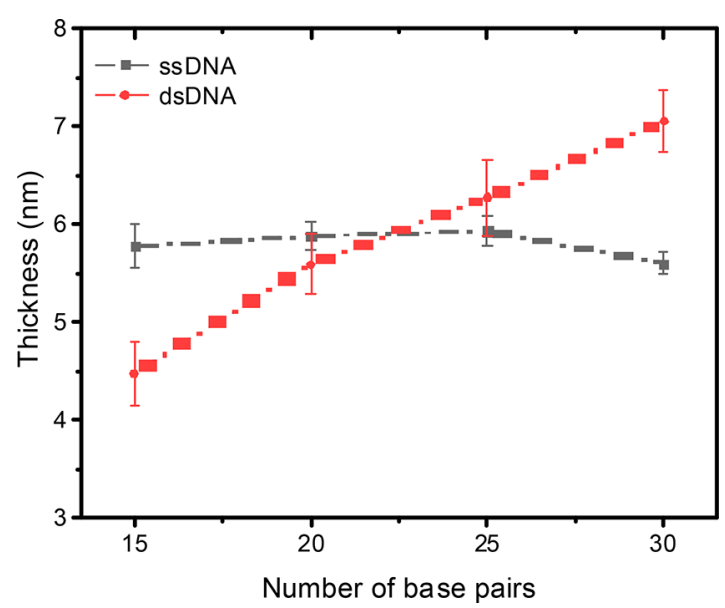

Figure 3. Thickness of the ssDNA and dsDNA SAMs on Au as a function of $n$ determined with ellipsometry; error bars represent the standard deviation obtained from 3-5 independent measurements on different samples. The dashed lines are visual guides.

of $n$. We observe that, for ssDNA, the thickness does not vary as a function of $n$, suggesting that the ssDNA SAMs suffer from significant disorder, in agreement with the $\mathrm{CV}$ results described earlier and in literature reports. ${ }^{37,56}$ In contrast, for dsDNA SAMs, we observe a monotonous increase in the thickness as a function of $n$. Interestingly, the thickness of the dsDNA $_{15}$ SAM is smaller than that of the ssDNA ${ }_{15}$ SAM. This observation can be explained since the formation of the
dsDNA helix upon hybridization reduces the length of the DNA fragments relative to the random coil structure adopted by ssDNA. ${ }^{57}$ We conclude that ssDNA SAMs on $\mathrm{Au}$ are disordered $^{16,17,37,39,40}$ and hybridization induces order due to the formation of the $\pi$-stack, in agreement with findings reported by others. ${ }^{17}$

$J(V)$ Characterization for ssDNA and dsDNA SAMs. We performed length-dependent $J(V)$ measurements for the

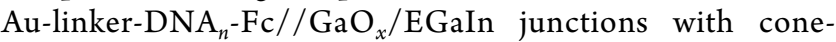
shaped EGaIn tips as a top-contact as a function of the number of base pairs indicated by the subscript $n$. The fabrication of the cone-tip junctions, statistical data collection, and data analysis were performed following previously reported procedures (see section S3.8 for details). ${ }^{58}$ Figures S14 and S15 show the histograms of $\log |J|$ at $\pm 1.0 \mathrm{~V}$ for all junctions along with a Gaussian fit to these histograms to determine the Gaussian average of $\log |J|\left(\langle\log |J|\rangle_{\mathrm{G}}\right)$, the Gaussian logstandard deviation $\left(\sigma_{\log , \mathrm{G}}\right)$, and the $95 \%$ confidence levels. Figure $4 \mathrm{~A}$ and B shows the corresponding $\langle\log |J|\rangle_{\mathrm{G}}$ vs $V$ curves which were measured at ambient conditions $(T=298 \mathrm{~K})$. Figure $4 \mathrm{C}$ shows that the value of $\langle\log |J|\rangle_{\mathrm{G}}$ at $-1.0 \mathrm{~V}$ as a function of $n$ for junctions with ssDNA does not follow a clear trend. In contrast, Figure 4D shows the same results but for junctions with dsDNA where $\langle\log |J|\rangle_{\mathrm{G}}$ follows a clear exponential decay with $n$. Fitting the data to eq 1 yields $\beta=$ $0.43 \pm 0.02 \mathrm{~nm}^{-1}$. The small observed asymmetry in the $J(V)$ curves is attributed to the $\mathrm{GaO}_{x}$ layer or other asymmetries present in the junctions. ${ }^{59}$ This rather different behavior can be explained by the results described above indicating that ssDNA forms disordered monolayers that fold back on themselves while the dsDNA monolayers are ordered. Therefore, the

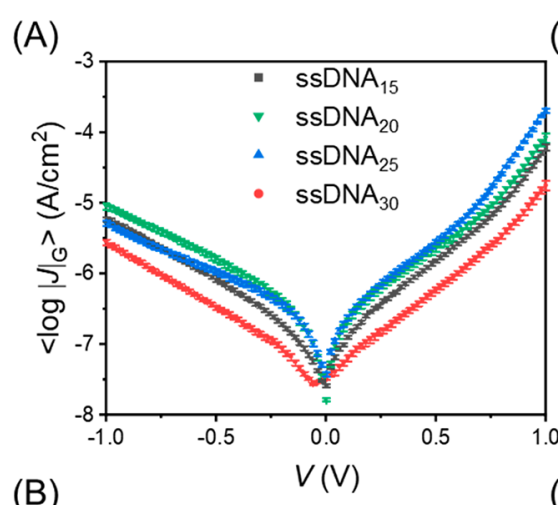

(C)
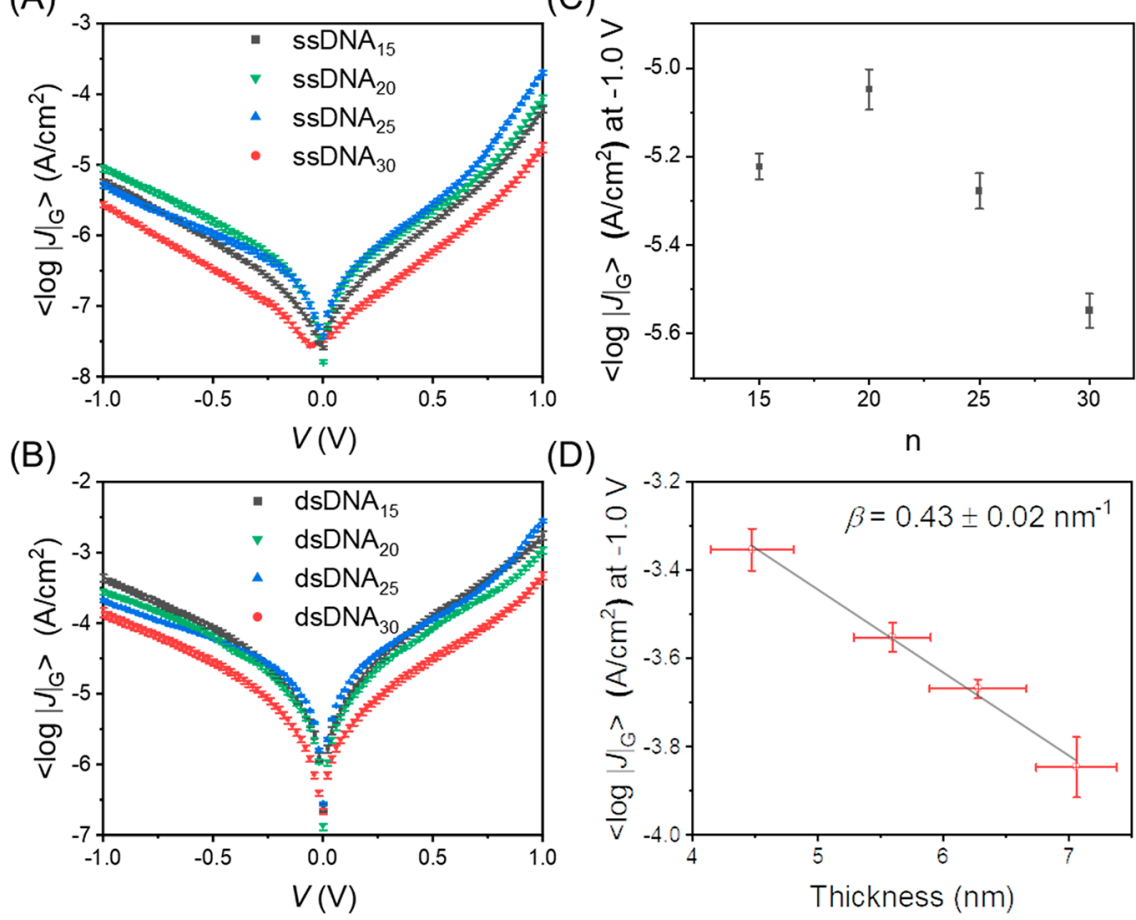

(D)

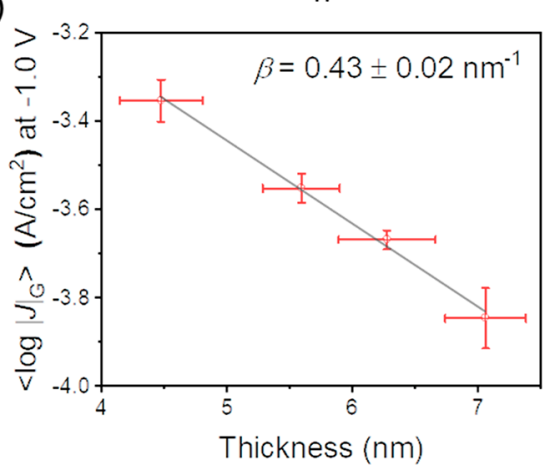

Figure 4. Plots of $\langle\log |J|\rangle_{\mathrm{G}}$ vs $V$ for (A) Au-linker-ssDNA-Fc//GaO $/$ EGaIn and (B) Au-linker-dsDNA-Fc//GaO $/$ /EGaIn. Error bars represent $95 \%$ confidence bands. (C) Plot of $\langle\log |J|\rangle_{\mathrm{G}}$ at $-1.0 \mathrm{~V}$ vs the number of ssDNA base pairs and (D) plot of $\langle\log |J|\rangle_{\mathrm{G}}$ at $-1.0 \mathrm{~V}$ vs ellipsometry thickness of Au-linker-dsDNA-Fc SAM. The solid line represents a fit to eq 1, $y$-axis error bars represent the $95 \%$ confidence intervals. $x$-Axis error bars represent the standard deviation for 3-5 independent measurements. All measurements are recorded at ambient temperature $(T=298 \mathrm{~K})$. 

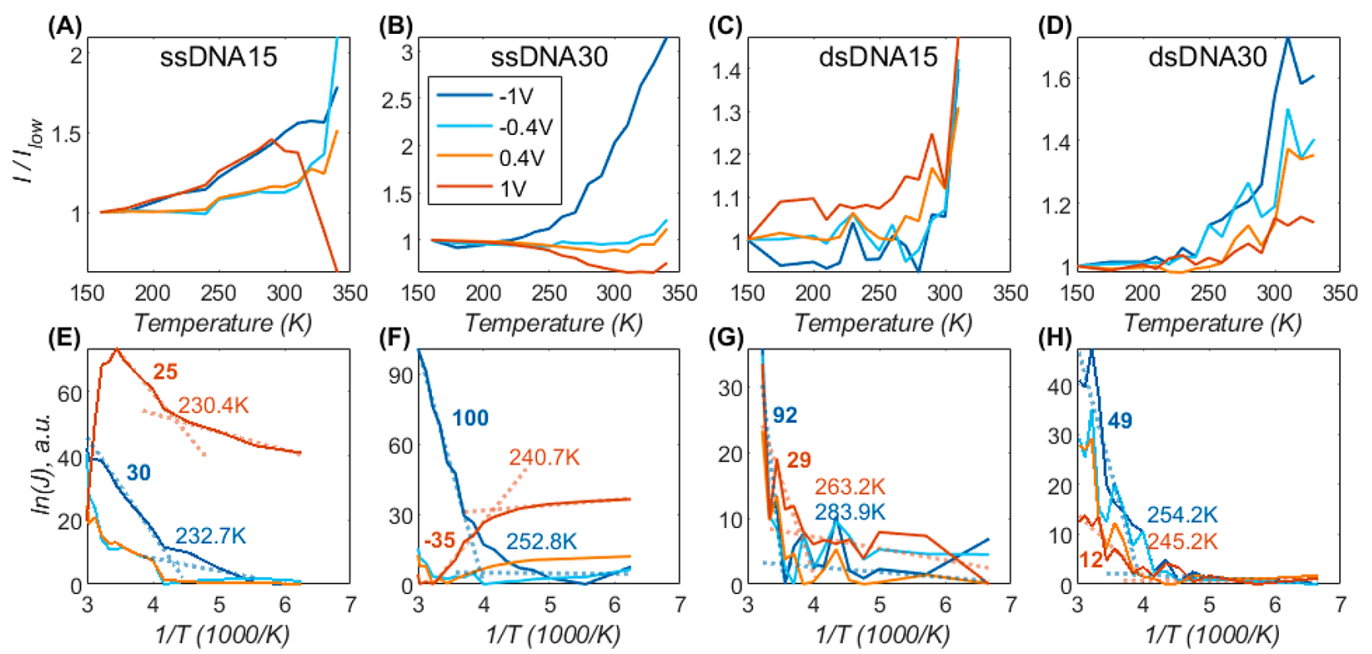

Figure 5. Variation of current with temperature on a linear scale $(\mathrm{A}-\mathrm{D})$ and as an Arrhenius plot $(\mathrm{E}-\mathrm{H})$, for shortest and longest Au-linker-DNA ${ }^{-}$ $\mathrm{Fc} / / \mathrm{GaO}_{x} / \mathrm{EGaIn}$ tunnel junctions, consisting of $\operatorname{ssDNA}_{15}(\mathrm{~A}, \mathrm{E}), \operatorname{ssDNA}_{30}(\mathrm{~B}, \mathrm{~F}), \mathrm{dsDNA}_{15}(\mathrm{C}, \mathrm{G})$, and dsDNA 30 (D, H). Each trace shows the current at a fixed voltage over a temperature range of $(340$ to $160 \mathrm{~K})$; for clarity, only four voltage values are shown (see legend in panel B). The entire data set is given in section S3.9. To emphasize temperature variations (cf. much larger voltage effect), the linear plots (A-D) show the current divided by its low $T$ value $\left(I(T) / I_{T \min }\right)$, and therefore, all traces start at 1 . Similarly, $\ln (J)$ traces $(\mathrm{E}-\mathrm{H})$ are arbitrarily vertically shifted and factorized to give slope units in $\mathrm{meV}(\Delta Y / \Delta X$ corresponds to $1 \mathrm{meV}$ slope). The $\pm 1 \mathrm{~V}$ traces were twice-fitted to eq 2 for high and low temperatures, separately (dotted lines, $\mathrm{E}-\mathrm{H}$ ); the crossing of the two lines is the transition temperature, $T_{\mathrm{C}}$, stated in each panel. The extracted slope value $\left(\sim E_{\mathrm{a}}\right.$, in $\left.\mathrm{meV}\right)$ of the high $T$ range is also given in the plots. The same raw data is used for the top and bottom panels; each current value is the average of 4 values measured during two consecutive $0 \mathrm{~V} \rightarrow+1 \mathrm{~V} \rightarrow-1 \mathrm{~V} \rightarrow 0 \mathrm{~V}$ loop-scans at a fixed temperature.
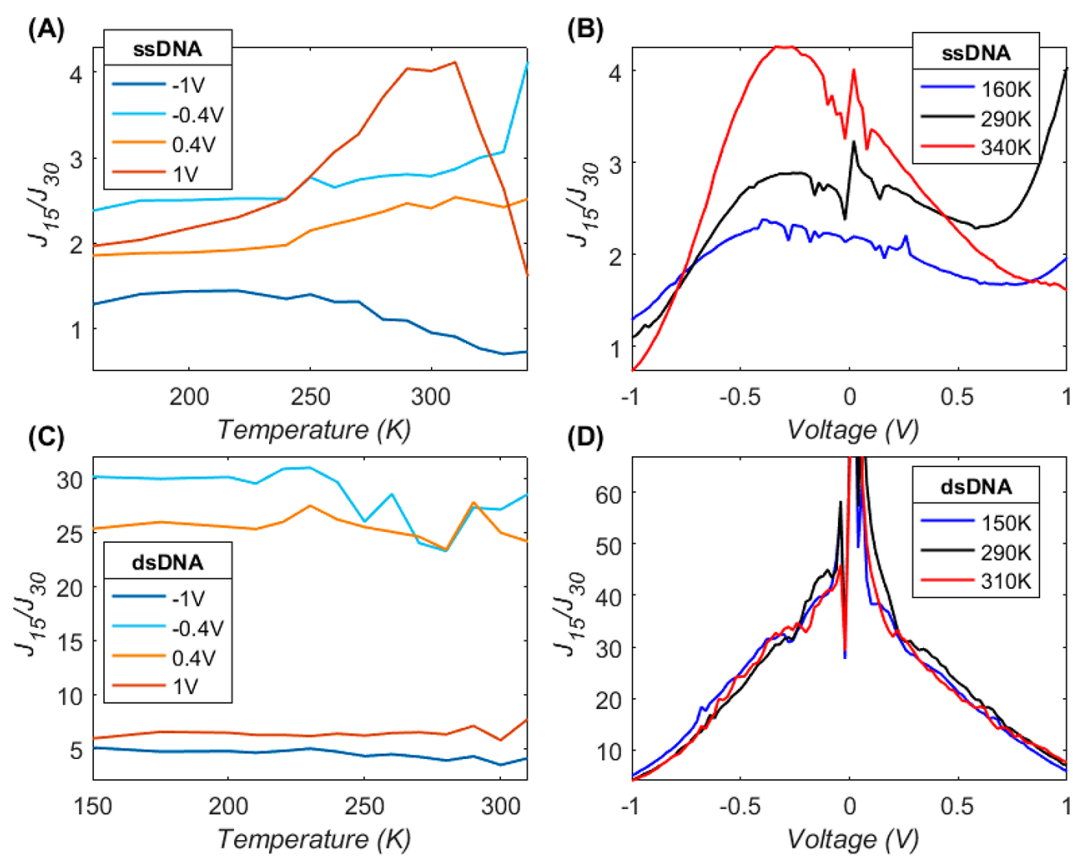

Figure 6. $J$ length attenuation for ssDNA $(\mathrm{A}, \mathrm{B})$ and dsDNA $(\mathrm{C}, \mathrm{D})$, showing the $J_{15} / J_{30}$ ratio between the current over 15-long relative to 30 -long bases (base-pairs): $(\mathrm{A}, \mathrm{C})$ as a function of temperature $(X$-axis) at four selected voltage values and $(\mathrm{B}, \mathrm{D})$ as a function of voltage $(X$-axis $)$ at three selected temperature values.

values of $\langle\log |J|\rangle_{\mathrm{G}}$ determined with ssDNA junctions lack a clear length dependency.

By comparing the $J(V)$ characteristics of ssDNA and dsDNA for identical values of $n$, we find that dsDNA is more conductive than ssDNA. For instance, values of $J$ for dsDNA at $V=-1.0 \mathrm{~V}$ are $31-74$ times larger than those values of $J$ for ssDNA. This finding is in agreement with previous reports where a 25-40 times higher conductance of dsDNA than ssDNA has been reported across single-molecule tunnel junctions. $^{34}$
Temperature-Dependent $J(V, T)$ Measurements. To investigate the mechanism of CT across the DNA junctions, we formed junctions with EGaIn stabilized in polymer-based microchannels perpendicularly aligned over Au strips supporting SAMs of the DNA that are described in detail in the Supporting Information (see section S3.9). As explained above, coherent (eq 1) and incoherent tunneling (eq 2) are characterized by distinctly different temperature, length, and voltage dependencies. To elucidate the dominant $\mathrm{CT}$ mechanism for each junction type, we performed $J(V, T)$ 
measurements for the two extreme lengths $(n=15$ and 30$)$ for both ssDNA- and dsDNA-based junctions across $T=150-340$ $\mathrm{K}$, though the $T$ ranges vary for dsDNA due to the calculated melting of dsDNA 15 at $312 \mathrm{~K}$ and of $\mathrm{dsDNA}_{30}$ at $335 \mathrm{~K}$, above which temperatures the junction conductance changed significantly (see the Supporting Information for details of melting point calculations). Figures 5 and $6 \mathrm{~A}$ show a summary of this analysis and the full data set is given in section S3.9). Figure 5A-D shows the $J$ normalized with the $J$ measured at the lowest $T$ at which CT was measured. The effect of $T$ on $J$ is significantly larger across junctions of ssDNA (Figure 5A,B, 23 -fold) than for dsDNA (Figure 5C,D, <2-fold). The effect of $T$ on $J$ also depends on $n$ : junctions with $n=30$ are more sensitive to $T$ than those junctions with $n=15$. For instance, junctions with $\mathrm{ssNA}_{30}$ (Figure $5 \mathrm{~B}$ ) were the most temperature-sensitive junctions while $\mathrm{CT}$ rates across dsDNA 15 (Figure 5C) are practically $T$-independent (except for minute temperature-enhancement at positive voltage). It is evident that the temperature effect is active only at high $T$, while CT rates are practically constant at low $T$, as was previously observed in protein junctions. ${ }^{12,60}$ This behavior can be qualitatively assigned to a transition from activationless tunneling (eq 1 ) at low $T$ to temperature-activated hopping (eq 2) at elevated $T$.

Another prominent observation from Figure 5 is that the $T$ effect changes as a function of the applied $V$. This effect is most prominent for ssDNA 30 (Figure $5 \mathrm{~B}$ ), where the $T$-effect is even inverted for opposite bias polarity: CT rates increase with $T$ for negative $V$ but decrease at positive $V$ and have a minimal effect at moderate $V$. For dsDNA 30 (Figure 5D), the $\mathrm{CT}$ rates increase with increasing $T$ at any $V$, yet the net change decreases with positive $V$ (the $+1 \mathrm{~V}$ (red) trace in Figure $5 \mathrm{D}$ is the lowest). Thus, these two junctions follow a similar qualitative trend. In contrast, junctions with ssDNA $\mathrm{N}_{15}$ (Figure $5 \mathrm{~A})$ show the most complicated behavior where the CT rates generally increase with $T$ at a rate proportional to $V$, except for the highest $V(+1 \mathrm{~V}$, red trace, Figure $5 \mathrm{~A})$, where this trend abruptly inverts at $\sim 300 \mathrm{~K}$.

To elucidate in more detail how both $V$ and $T$ affect the CT rates, we analyzed the Arrhenius plots of the same data (Figure $5 \mathrm{E}-\mathrm{H}$ ) where the $\ln J$ traces are vertically shifted to align with their minimal values to focus on the $T$ effect (cf. $V$ ). To identify the transition temperature, $T_{\mathrm{C}}$, between activationless to activated CT, we fitted the data to eq 2 over high and low $T$ values and assigned the crossing of the two linear fits to $T_{\mathrm{C}}{ }^{12}$ The extracted $T_{\mathrm{C}}$ values (see in-plot labels) are between 230 and $280 \mathrm{~K}$ and are slightly higher than what was observed in junctions of azurin $(\sim 200 \mathrm{~K}))^{12,60}$ Considering the total $J$ as a summation of tunneling and hopping channels (eq 3), a higher $T_{\mathrm{C}}$ implies that coherent tunneling persists up to higher $T$ : for ssDNA $T_{\mathrm{C}} \cong 235 \mathrm{~K}$ compared to $\sim 260 \mathrm{~K}$ for dsDNA. Incoherent tunneling is also less likely under negative bias polarity as $T_{\mathrm{C}}(-1 \mathrm{~V}) \approx T_{\mathrm{C}}(+1 \mathrm{~V})+10 \mathrm{~K}$. The interplay between $T$ and $V$ effects on the $\mathrm{CT}$ rates implies that an applied $V$ modulates the $E_{\text {a }}$ of eq 2 . There are two principal scenarios for the $V$ effect: it can (a) shift the energy alignment between the molecule and the electrodes or (b) reduce the energy gap between neighbor hopping sites along the molecular skeleton; hopping over the contact (a) is expected to be asymmetric and preserve the $V$ polarity, while the $V$ effect on hopping along the molecular skeleton (b) is facilitated by the absolute electric field, regardless of voltage polarity.
The net effective $E_{\mathrm{a}}$ can be extracted from the slope of the Arrhenius plot:

$$
E_{\mathrm{a}, \mathrm{eff}}=-\left.k_{\mathrm{B}} \frac{\Delta \ln J}{\Delta T}\right|_{V}=\left\{\begin{array}{c}
E_{\mathrm{a}}-\delta V \\
E_{\mathrm{a}}-\delta|V|
\end{array}\right.
$$

where the effective $E_{\mathrm{a} \text {,eff }}$ is the summation of an intrinsic value, $E_{a}$ and $V$ dependency, $\delta V$, either preserving polarity (top equation of 4 , contact-hopping) or an absolute effect (bottom equation of 4, multiple-sites hopping). The $\delta$ factor reflects the partial $V$ drop on each hopping site: for multiple hopping over $N$-consecutive sites, $\delta \sim \frac{1}{N},|V|$, while for a single electrode to the molecule hopping $\delta$ is the fractional voltage drop on the hopping-interface.

The $E_{\mathrm{a}, \text { eff }}$ values extracted from fit slopes (high $T$ only) are shown as labels in Figure $5 \mathrm{E}-\mathrm{H}$. They show that $E_{\mathrm{a} \text { eff }}$ decreases as the $V$ becomes more positive in all cases except ssDNA $_{15}$ (Figure 5E). A full $E_{\mathrm{a}, \text { eff }}$ analysis is given in section S3.9. The reduction in $E_{\mathrm{a}, \text { eff }}$ was most prominent for $\mathrm{ssDNA}_{30}$ (Figure $5 \mathrm{~F}$ and section S3.9), where $E_{\mathrm{a} \text { eff }}$ becomes negative (observed positive slope), providing a strong indication that the hopping (or $T$-activated step) relates to the injection of carriers from the electrode into the DNA and not to CT along with the $\sim 5 \mathrm{~nm}$ thick DNA film. The shortest ssDNA $\left(\right.$ ssDNA $_{15}$, Figure 5E and section S3.9) is the only DNA strand where $E_{\mathrm{a} \text {,eff }}$ increased with $|V|$ for both bias polarities, i.e., against the trivial expectation that an external field reduces the $E_{\mathrm{a}, \mathrm{eff}}$. However, the net $E_{\mathrm{a}, \text { eff }}$ values for this DNA were not high (15-40 meV), and the behavior becomes even more complicated at $+1 \mathrm{~V}$ (red trace) with a second transition into an inverted dependency. The extracted $E_{\mathrm{a} \text {,eff }}$ values confirm the qualitative observation that $\mathrm{CT}$ across $\mathrm{ssDNA}_{30}$ is the most "hopping-dominated" of DNA junctions investigated, with an $E_{\mathrm{a}, \text { eff }}$ reaching $100 \mathrm{meV}$. The junctions of ssDNA $_{15}$ and $\mathrm{dsDNA}_{30}$ exist in the intermediate regime with $E_{\text {a,eff }}$ up to $\sim 40 \mathrm{meV}$, and the dsDNA 15 (Figure 5G) does show high slopes but they are limited to a very small $T$-range and, therefore, could reflect noise. Inserting $E_{\mathrm{a}, \text { eff }}$ values (see the Supporting Information for complete set) in eq 4, we can estimate the equilibrium $E_{\mathrm{a}} \simeq 30-60 \mathrm{meV}$ and $\delta \simeq 0.02-0.07$.

Figure 5 and eq 4 show that the value of $E_{\mathrm{a}}$ increases with $V$ as a function of the polarity. We have reported a similar behavior in three-terminal single-molecule devices containing redox-active molecules with ferrocene moieties in the Coulomb blockade regime. ${ }^{61}$ For these reasons, we suggest that the DNA junctions transition from a regime that resembles a resonant tunneling regime to a regime that most likely resembles Coulomb blockade (where charges reside on the molecule, or hopping regime), where the current increases exponentially with the $T$. Alternatively, this kind of anomalous behavior has also been observed in junctions transiting into the inverted Marcus regime. ${ }^{62}$ The temperature-independent CT in junctions with double-stranded DNA agrees with former findings by van Zalinge et al., ${ }^{41}$ albeit over a smaller $T$ range of 295-333 K and fixed $0.2 \mathrm{~V}$ voltage.

Length Dependence of $J(V, T)$ Measurements. As explained in the Introduction, the length $(d$, in $\mathrm{nm})$ is another aspect that helps us to distinctly differentiate between incoherent and coherent tunneling CT. Coherent tunneling has a strong exponential dependence on $d$. Figure 6 shows the $d$ dependence of DNA tunnel junctions by taking the ratio between $J$ across junctions with short DNA and $J$ across 
junctions with long DNA: $J\left(\operatorname{ssDNA}_{15}\right) / J\left(\operatorname{ssDNA}_{30}\right)$ in Figure $6 \mathrm{~A}, \mathrm{~B}$ and $J\left(\mathrm{dsDNA}_{15}\right) / J\left(\mathrm{dsDNA}_{30}\right)$ in Figure $6 \mathrm{C}, \mathrm{D}$. The comparison is performed as a function of $T$ at a few selected $V$ values (Figure 6A,C) and as a function of $V$ at a few selected isotherms (Figure 6B,D). The dominance of tunneling for dsDNA is clear by up to 30 -fold length attenuation (Figure $6 \mathrm{C}$ ), and the length attenuation (or tunneling behavior) is stronger at low voltage $(V< \pm 0.4 \mathrm{~V})$ than at high voltages $(V$ $= \pm 1 \mathrm{~V}$ with only a 5-7-fold attenuation). This is in perfect agreement with the $T$ effect (Figure 5). We observe that, at low $V$ (or low $T$ ), the $J$ is small and CT is dominated by tunneling processes. As the $V$ increases (or at high $T$ ), the tunneling alone cannot transport the high $J$ and other mechanisms, like hopping, become dominant.

We have not attempted to translate the current ratio into the tunneling decay coefficient, $\beta$, because of only two data points, though in principle we expect $\beta=\ln \left(J_{15} / J_{30}\right) / \Delta d$. The voltage dependence of the current ratio of dsDNA (Figure 6D) shows the classical expected decay of $\beta$ ( $J$ ratio) with voltage. ${ }^{63-67}$ The clear voltage dependence (Figure 6D) is in stark contrast to the lack of temperature dependence (Figure 6C) which is exactly expected from a tunneling process. The $d$ dependency for ssDNA (Figure 6A,B) was much weaker, up to 4-fold at maximum. This observation strengthens our conclusion that CT across ssDNA is hopping-dominated. However, there are still many unresolved questions in our studies, for example, the $T$ dependence (Figure 5) suggests that, at low $T$, both ssDNA and dsDNA undergo $\mathrm{CT}$ by an activationless tunneling mechanism. In contrast, Figure $6 \mathrm{~A}$ shows hardly any $d$ attenuation at these low $T$ points. In section S3.9, we show all the measured $J(V, T)$ data, which also reveals this complex behavior. This suggests that the prevailing identity between temperature-inactivated and "tunneling" is probably an oversimplification, and more studies are needed to reveal more details regarding the $\mathrm{CT}$ mechanism. ${ }^{12,67}$

\section{CONCLUSIONS}

We have confirmed that dsDNA is at least 30 -fold more conductive than ssDNA in large-area tunnel junctions based on EGaIn. On the basis of CV and ellipsometry, we demonstrate that ssDNA forms disordered monolayers that undergo backbending on Au electrodes. On the basis of our $J(V, T)$ studies, we conclude that thermally activated hopping is the mechanism of $\mathrm{CT}$ in the junctions with disordered ssDNA for $T=240-330 \mathrm{~K}$. At low $T(T<240 \mathrm{~K})$, the contribution from the thermally activated hopping can be neglected and (activationless) coherent tunneling dominates CT. Interchain hopping limits charge conduction in ssDNA due to which CT rates are comparatively low as the charge has to "hop" from one chain to another. On the other hand, CT rates across dsDNA are high because dsDNA forms ordered monolayers that possess a $\pi$-stack and displays CT behavior in which charge can readily coherently tunnel along the chain (but a small thermally active component appears for large enough values of $d$ as also has been observed for other types of molecular wires ${ }^{68-72}$ ). Therefore, we conclude that intrachain coherent tunneling is a more efficient charge conduction channel than interchain hopping in DNA tunnel junctions, with our studies revealing the vital importance of structural order in developing efficient (bio)molecular electronics devices.

\section{ASSOCIATED CONTENT}

\section{Supporting Information}

The Supporting Information is available free of charge at https://pubs.acs.org/doi/10.1021/jacs.1c09549.

Experimental details, synthesis, characterization, and purification of the DNA, self-assembly and characterization of DNA monolayers with AR-XPS, and CV for ellipsometry; statistical analysis of $J(V)$ measurements and temperature-dependent $J(V)$ measurements (PDF)

\section{AUTHOR INFORMATION}

\section{Corresponding Authors}

Ayelet Vilan - Department of Chemical and Biological Physics, Weizmann Institute of Science, Rehovot 76100, Israel; (1) orcid.org/0000-0001-5126-9315; Email: ayelet.vilan@weizmann.ac.il

Eimer M. Tuite - Chemistry-School of Natural and Environmental Sciences, Newcastle University, Newcastle upon Tyne NE1 7RU, United Kingdom; (1) orcid.org/00000002-2805-4858; Email: eimer.tuite@newcastle.ac.uk

Andrew R. Pike - Chemistry-School of Natural and Environmental Sciences, Newcastle University, Newcastle upon Tyne NE1 7RU, United Kingdom; (1) orcid.org/00000002-1888-9798; Email: andrew.pike@newcastle.ac.uk

James H. R. Tucker - School of Chemistry, University of Birmingham, Birmingham, West Midlands B15 2TT, United Kingdom; 이이이.org/0000-0001-7645-0815;

Email: j.tucker@bham.ac.uk

Christian A. Nijhuis - Department of Chemistry, National University of Singapore, Singapore 117543, Singapore; Centre for Advanced 2D Materials, National University of Singapore, Singapore 117546, Singapore; Department of Molecules \& Materials, MESA+ Institute for Nanotechnology, Faculty of Science and Technology, University of Twente, 7500 AE Enschede, The Netherlands; 이이.org/00000003-3435-4600; Email: c.a.nijhuis@utwente.nl

\section{Authors}

Nipun Kumar Gupta - Department of Chemistry, National University of Singapore, Singapore 117543, Singapore; Centre for Advanced 2D Materials, National University of Singapore, Singapore 117546, Singapore

Edward A. Wilkinson - School of Chemistry, University of Birmingham, Birmingham, West Midlands B15 2TT, United Kingdom; (1) orcid.org/0000-0002-4987-6965

Senthil Kumar Karuppannan - Department of Chemistry, National University of Singapore, Singapore 117543, Singapore

Lily Bailey - School of Chemistry, University of Birmingham, Birmingham, West Midlands B15 2TT, United Kingdom

Ziyu Zhang - Department of Chemistry, National University of Singapore, Singapore 117543, Singapore

Dong-Chen Qi - Centre for Materials Science, School of Chemistry and Physics, Queensland University of Technology, Brisbane, Queensland 4001, Australia; orcid.org/00000001-8466-0257

Anton Tadich - Australian Synchrotron Clayton, Clayton,

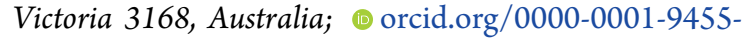
5901

Complete contact information is available at:

https://pubs.acs.org/10.1021/jacs.1c09549 


\section{Author Contributions}

II N.K.G. and E.A.W. contributed equally.

\section{Notes}

The authors declare no competing financial interest.

\section{ACKNOWLEDGMENTS}

Dr. Bruce Cowie and Australian Synchrotron, and the Singapore Synchrotron Light Source are acknowledged for assistance with the photoelectron spectroscopy measurements. Funding by the Ministry of Education (MOE) for supporting this research under Award No. MOE2019-T2-1-137 and R143-000-B30-112 are acknowledged. Prime Minister's Office, Singapore under its Medium sized centre program is also acknowledged for supporting this research. The Centre for Chemical and Materials Analysis in the School of Chemistry at the University of Birmingham is acknowledged for technical support. This project has received funding from the European Union's Horizon 2020 research and innovation programme under the Marie Skłodowska-Curie Grant Agreement No. 778001 .

\section{REFERENCES}

(1) Endres, R. G.; Cox, D. L.; Singh, R. R. P. Colloquium: The Quest for High-Conductance DNA. Rev. Mod. Phys. 2004, 76 (1), 195-214.

(2) Eley, D. D.; Spivey, D. I. Semiconductivity of Organic Substances. Part 9.-Nucleic Acid in the Dry State. Trans. Faraday Soc. 1962, 58, 411-415.

(3) Chen, Z.; Kibler, R. D.; Hunt, A.; Busch, F.; Pearl, J.; Jia, M.; VanAernum, Z. L.; Wicky, B. I. M. M.; Dods, G.; Liao, H.; Wilken, M. S.; Ciarlo, C.; Green, S.; El-Samad, H.; Stamatoyannopoulos, J.; Wysocki, V. H.; Jewett, M. C.; Boyken, S. E.; Baker, D. De Novo Design of Protein Logic Gates. Science (Washington, DC, U. S.) 2020, 368 (6486), 78-84.

(4) Wang, Y.; Yan, X.; Dong, R. Organic Memristive Devices Based on Silver Nanoparticles and DNA. Org. Electron. 2014, 15 (12), 3476-3481.

(5) Lakhno, V. D. DNA Nanobioelectronics. Int. J. Quantum Chem. 2008, 108 (11), 1970-1981.

(6) Hung, Y.-C.; Hsu, W.-T.; Lin, T.-Y.; Fruk, L. Photoinduced Write-Once Read-Many-Times Memory Device Based on DNA Biopolymer Nanocomposite. Appl. Phys. Lett. 2011, 99 (25), 253301.

(7) Guo, C.; Wang, K.; Zerah-Harush, E.; Hamill, J.; Wang, B.; Dubi, Y.; Xu, B. Molecular Rectifier Composed of DNA with High Rectification Ratio Enabled by Intercalation. Nat. Chem. 2016, 8 (5), 484-490.

(8) Xiang, L.; Palma, J. L.; Li, Y.; Mujica, V.; Ratner, M. A.; Tao, N. Gate-Controlled Conductance Switching in DNA. Nat. Commun. 2017, $8,1-10$.

(9) Thompson, D.; Nijhuis, C. A. Even the Odd Numbers Help: Failure Modes of SAM-Based Tunnel Junctions Probed via Odd-Even Effects Revealed in Synchrotrons and Supercomputers. Acc. Chem. Res. 2016, 49 (10), 2061-2069.

(10) Chen, X.; Hu, H.; Trasobares, J.; Nijhuis, C. A. Rectification Ratio and Tunneling Decay Coefficient Depend on the Contact Geometry Revealed by in Situ Imaging of the Formation of EGaIn Junctions. ACS Appl. Mater. Interfaces 2019, 11 (23), 21018-21029.

(11) Sepunaru, L.; Friedman, N.; Pecht, I.; Sheves, M.; Cahen, D. Temperature-Dependent Solid-State Electron Transport through Bacteriorhodopsin: Experimental Evidence for Multiple Transport Paths through Proteins. J. Am. Chem. Soc. 2012, 134 (9), 4169-4176.

(12) Valianti, S.; Cuevas, J. C.; Skourtis, S. S. Charge-Transport Mechanisms in Azurin-Based Monolayer Junctions. J. Phys. Chem. C 2019, 123 (10), 5907-5922.
(13) Shi, H.; Yang, F.; Li, W.; Zhao, W.; Nie, K.; Dong, B.; Liu, Z. A Review: Fabrications, Detections and Applications of Peptide Nucleic Acids (PNAs) Microarray. Biosens. Bioelectron. 2015, 66, 481-489.

(14) Venkatramani, R.; Keinan, S.; Balaeff, A.; Beratan, D. N. Nucleic Acid Charge Transfer: Black, White and Gray. Coord. Chem. Rev. 2011, 255 (7-8), 635-648.

(15) Giese, B.; Amaudrut, J.; Köhler, A.-K.; Spormann, M.; Wessely, S. Direct Observation of Hole Transfer through DNA by Hopping between Adenine Bases and by Tunnelling. Nature 2001, 412 (6844), 318-320.

(16) Paul, A.; Watson, R. M.; Lund, P.; Xing, Y.; Burke, K.; He, Y.; Borguet, E.; Achim, C.; Waldeck, D. H. Charge Transfer through Single-Stranded Peptide Nucleic Acid Composed of Thymine Nucleotides. J. Phys. Chem. C 2008, 112 (18), 7233-7240.

(17) Paul, A.; Watson, R. M.; Wierzbinski, E.; Davis, K. L.; Sha, A.; Achim, C.; Waldeck, D. H. Distance Dependence of the Charge Transfer Rate for Peptide Nucleic Acid Monolayers †. J. Phys. Chem. B 2010, 114 (45), 14140-14148.

(18) Venkatramani, R.; Davis, K. L.; Wierzbinski, E.; Bezer, S.; Balaeff, A.; Keinan, S.; Paul, A.; Kocsis, L.; Beratan, D. N.; Achim, C.; Waldeck, D. H. Evidence for a Near-Resonant Charge Transfer Mechanism for Double-Stranded Peptide Nucleic Acid. J. Am. Chem. Soc. 2011, 133 (1), 62-72.

(19) Boon, E. M.; Barton, J. K. Charge Transport in DNA. Curr. Opin. Struct. Biol. 2002, 12 (3), 320-329.

(20) Livshits, G. I.; Stern, A.; Rotem, D.; Borovok, N.; Eidelshtein, G.; Migliore, A.; Penzo, E.; Wind, S. J.; Di Felice, R.; Skourtis, S. S.; Cuevas, J. C.; Gurevich, L.; Kotlyar, A. B.; Porath, D. Long-Range Charge Transport in Single G-Quadruplex DNA Molecules. Nat. Nanotechnol. 2014, 9 (12), 1040-1046.

(21) Xiang, L.; Palma, J. L.; Bruot, C.; Mujica, V.; Ratner, M. A.; Tao, N. Intermediate Tunnelling-Hopping Regime in DNA Charge Transport. Nat. Chem. 2015, 7 (3), 221-226.

(22) Anne, A.; Bouchardon, A.; Moiroux, J. 3'-Ferrocene-Labeled Oligonucleotide Chains End-Tethered to Gold Electrode Surfaces: Novel Model Systems for Exploring Flexibility of Short DNA Using Cyclic Voltammetry. J. Am. Chem. Soc. 2003, 125 (5), 1112-1113.

(23) Fan, C.; Plaxco, K. W.; Heeger, A. J. Electrochemical Interrogation of Conformational Changes as a Reagentless Method for the Sequence-Specific Detection of DNA. Proc. Natl. Acad. Sci. U. S. A. 2003, 100 (16), 9134-9137.

(24) Hasegawa, Y.; Takada, T.; Nakamura, M.; Yamana, K. Ferrocene Conjugated Oligonucleotide for Electrochemical Detection of DNA Base Mismatch. Bioorg. Med. Chem. Lett. 2017, 27 (15), $3555-3557$

(25) Kang, D.; Zuo, X.; Yang, R.; Xia, F.; Plaxco, K. W.; White, R. J. Comparing the Properties of Electrochemical-Based DNA Sensors Employing Different Redox Tags. Anal. Chem. 2009, 81 (21), 91099113.

(26) Silva, S. M.; Hoque, S.; Gonçales, V. R.; Gooding, J. J. The Impact of the Position of the Redox Label on Charge Transfer and Hybridization Efficiency at DNA Interfaces. Electroanalysis 2018, 30 (7), 1529-1535.

(27) Slinker, J. D.; Muren, N. B.; Renfrew, S. E.; Barton, J. K. DNA Charge Transport over $34 \mathrm{Nm}$. Nat. Chem. 2011, 3 (3), 228-233.

(28) Liu, T.; Barton, J. K. DNA Electrochemistry through the Base Pairs Not the Sugar-Phosphate Backbone. J. Am. Chem. Soc. 2005, 127 (29), 10160-10161.

(29) Zwang, T. J.; Tse, E. C. M. M.; Barton, J. K. Sensing DNA through DNA Charge Transport. ACS Chem. Biol. 2018, 13 (7), 1799-1809.

(30) van Zalinge, H.; Schiffrin, D. J.; Bates, A. D.; Haiss, W.; Ulstrup, J.; Nichols, R. J. Single-Molecule Conductance Measurements of Single- and Double-Stranded DNA Oligonucleotides. ChemPhysChem 2006, 7 (1), 94-98.

(31) Cohen, H.; Nogues, C.; Ullien, D.; Daube, S.; Naaman, R.; Porath, D. Electrical Characterization of Self-Assembled Single- and Double-Stranded DNA Monolayers Using Conductive AFM. Faraday Discuss. 2006, 131, 367-376. 
(32) Cohen, H.; Nogues, C.; Naaman, R.; Porath, D. Direct Measurement of Electrical Transport through Single DNA Molecules of Complex Sequence. Proc. Natl. Acad. Sci. U. S. A. 2005, 102 (33), 11589-11593.

(33) Tsai, C.-Y.; Chang, T.-L.; Kuo, L.-S.; Chen, P.-H. Detection of Electrical Characteristics of DNA Strands Immobilized on SelfAssembled Multilayer Gold Nanoparticles. Appl. Phys. Lett. 2006, 89 (20), 203902.

(34) Roy, S.; Vedala, H.; Roy, A. D.; Kim, D.; Doud, M.; Mathee, K.; Shin, H.; Shimamoto, N.; Prasad, V.; Choi, W. Direct Electrical Measurements on Single-Molecule Genomic DNA Using SingleWalled Carbon Nanotubes. Nano Lett. 2008, 8 (1), 26-30.

(35) Guo, X.; Gorodetsky, A. A.; Hone, J.; Barton, J. K.; Nuckolls, C. Conductivity of a Single DNA Duplex Bridging a Carbon Nanotube Gap. Nat. Nanotechnol. 2008, 3 (3), 163-167.

(36) Zhuravel, R.; Huang, H.; Polycarpou, G.; Polydorides, S.; Motamarri, P.; Katrivas, L.; Rotem, D.; Sperling, J.; Zotti, L. A.; Kotlyar, A. B.; Cuevas, J. C.; Gavini, V.; Skourtis, S. S.; Porath, D. Backbone Charge Transport in Double-Stranded DNA. Nat. Nanotechnol. 2020, 15 (10), 836-840.

(37) Paul, A.; Bezer, S.; Venkatramani, R.; Kocsis, L.; Wierzbinski, E.; Balaeff, A.; Keinan, S.; Beratan, D. N.; Achim, C.; Waldeck, D. H. Role of Nucleobase Energetics and Nucleobase Interactions in SingleStranded Peptide Nucleic Acid Charge Transfer. J. Am. Chem. Soc. 2009, 131 (18), 6498-6507.

(38) Steel, A. B.; Levicky, R. L.; Herne, T. M.; Tarlov, M. J. Immobilization of Nucleic Acids at Solid Surfaces: Effect of Oligonucleotide Length on Layer Assembly. Biophys. J. 2000, 79 (2), 975-981.

(39) Legay, G.; Markey, L.; Meunier-Prest, R.; Finot, E. Measurements of Thickness Dispersion in Biolayers by Scanning Force Microscopy and Comparison with Spectroscopic Ellipsometry Analysis. Ultramicroscopy 2007, 107 (10-11), 1111-1117.

(40) Legay, G.; Finot, E.; Meunier-Prest, R.; Cherkaoui-Malki, M.; Latruffe, N.; Dereux, A. DNA Nanofilm Thickness Measurement on Microarray in Air and in Liquid Using an Atomic Force Microscope. Biosens. Bioelectron. 2005, 21 (4), 627-636.

(41) van Zalinge, H.; Schiffrin, D. J.; Bates, A. D.; Starikov, E. B.; Wenzel, W.; Nichols, R. J. Variable-Temperature Measurements of the Single-Molecule Conductance of Double-Stranded DNA. Angew. Chem., Int. Ed. 2006, 45 (33), 5499-5502.

(42) Livshits, G. I.; Ghabboun, J.; Borovok, N.; Kotlyar, A. B.; Porath, D. Comparative Electrostatic Force Microscopy of Tetra- and Intra-Molecular G4-DNA. Adv. Mater. 2014, 26 (29), 4981-4985.

(43) Zhang, H.; Chao, J.; Pan, D.; Liu, H.; Huang, Q.; Fan, C. Folding Super-Sized DNA Origami with Scaffold Strands from LongRange PCR. Chem. Commun. 2012, 48 (51), 6405.

(44) Ihara, T.; Sasahara, D.; Shimizu, M.; Jyo, A. DNA Conjugates Bearing a Ferrocenyl Group in Backbone and Their Electrochemical Behaviour. Supramol. Chem. 2009, 21 (3-4), 207-217.

(45) Jiang, L.; Yuan, L.; Cao, L.; Nijhuis, C. A. Controlling Leakage Currents: The Role of the Binding Group and Purity of the Precursors for Self-Assembled Monolayers in the Performance of Molecular Diodes. J. Am. Chem. Soc. 2014, 136 (5), 1982-1991.

(46) Yuan, L.; Jiang, L.; Thompson, D.; Nijhuis, C. A. On the Remarkable Role of Surface Topography of the Bottom Electrodes in Blocking Leakage Currents in Molecular Diodes. J. Am. Chem. Soc. 2014, 136 (18), 6554-6557.

(47) Nerngchamnong, N.; Thompson, D.; Cao, L.; Yuan, L.; Jiang, L.; Roemer, M.; Nijhuis, C. A. Nonideal Electrochemical Behavior of Ferrocenyl-Alkanethiolate SAMs Maps the Microenvironment of the Redox Unit. J. Phys. Chem. C 2015, 119 (38), 21978-21991.

(48) Rowe, G. K.; Creager, S. E. Redox and Ion-Pairing Thermodynamics in Self-Assembled Monolayers. Langmuir 1991, 7 (10), 2307-2312.

(49) Duffin, T. J.; Nerngchamnong, N.; Thompson, D.; Nijhuis, C. A. Direct Measurement of the Local Field within Alkyl-FerrocenylAlkanethiolate Monolayers: Importance of the Supramolecular and
Electronic Structure on the Voltammetric Response and Potential Profile. Electrochim. Acta 2019, 311, 92-102.

(50) Rudnev, A. V.; Yoshida, K.; Wandlowski, T. Electrochemical Characterization of Self-Assembled Ferrocene-Terminated Alkanethiol Monolayers on Low-Index Gold Single Crystal Electrodes. Electrochim. Acta 2013, 87, 770-778.

(51) Kelley, S. O.; Barton, J. K.; Jackson, N. M.; Hill, M. G. Electrochemistry of Methylene Blue Bound to a DNA-Modified Electrode. Bioconjugate Chem. 1997, 8 (1), 31-37.

(52) Kumar, K. S.; Naaman, R. Quantitative Analysis and Characterization of Self-Assembled DNA on a Silver Surface. Langmuir 2012, 28 (41), 14514-14517.

(53) Domínguez, C. M.; Ramos, D.; Mendieta-Moreno, J. I.; Fierro, J. L. G.; Mendieta, J.; Tamayo, J.; Calleja, M. Effect of Water-DNA Interactions on Elastic Properties of DNA Self-Assembled Monolayers. Sci. Rep. 2017, 7 (1), 536.

(54) Lee, L. Y. S.; Sutherland, T. C.; Rucareanu, S.; Lennox, R. B. Ferrocenylalkylthiolates as a Probe of Heterogeneity in Binary SelfAssembled Monolayers on Gold. Langmuir 2006, 22 (9), 4438-4444.

(55) Vilar, M. R.; Botelho do Rego, A. M.; Ferraria, A. M.; Jugnet, Y.; Noguès, C.; Peled, D.; Naaman, R. Interaction of Self-Assembled Monolayers of DNA with Electrons: HREELS and XPS Studies. J. Phys. Chem. B 2008, 112 (23), 6957-6964.

(56) Paul, A.; Watson, R. M.; Lund, P.; Xing, Y.; Burke, K.; He, Y.; Borguet, E.; Achim, C.; Waldeck, D. H. Charge Transfer through Single-Stranded Peptide Nucleic Acid Composed of Thymine Nucleotides. J. Phys. Chem. C 2008, 112 (18), 7233-7240.

(57) Elhadj, S.; Singh, G.; Saraf, R. F. Optical Properties of an Immobilized DNA Monolayer from 255 to $700 \mathrm{Nm}$. Langmuir 2004, 20 (13), 5539-5543.

(58) Nerngchamnong, N.; Yuan, L.; Qi, D.-C.; Li, J.; Thompson, D.; Nijhuis, C. A. The Role of van Der Waals Forces in the Performance of Molecular Diodes. Nat. Nanotechnol. 2013, 8 (2), 113-118.

(59) Karuppannan, S. K.; Hongting, H.; Troadec, C.; Vilan, A.; Nijhuis, C. A. Ultrasmooth and Photoresist-Free Micropore-Based EGaIn Molecular Junctions: Fabrication and How Roughness Determines Voltage Response. Adv. Funct. Mater. 2019, 29 (38), 1904452.

(60) Amdursky, N.; Sepunaru, L.; Raichlin, S.; Pecht, I.; Sheves, M.; Cahen, D. Electron Transfer Proteins as Electronic Conductors: Significance of the Metal and Its Binding Site in the Blue $\mathrm{Cu}$ Protein. Azurin. Adv. Sci. 2015, 2 (4), 1400026.

(61) Garrigues, A. R.; Wang, L.; del Barco, E.; Nijhuis, C. A. Electrostatic Control over Temperature-Dependent Tunnelling across a Single-Molecule Junction. Nat. Commun. 2016, 7 (May), 11595.

(62) Yuan, L.; Wang, L.; Garrigues, A. R.; Jiang, L.; Annadata, H. V.; Anguera Antonana, M.; Barco, E.; Nijhuis, C. A. Transition from Direct to Inverted Charge Transport Marcus Regions in Molecular Junctions via Molecular Orbital Gating. Nat. Nanotechnol. 2018, 13 (4), 322-329.

(63) Cui, X. D.; Primak, A.; Zarate, X.; Tomfohr, J.; Sankey, O. F.; Moore, A. L.; Moore, T. A.; Gust, D.; Nagahara, L. A.; Lindsay, S. M. Changes in the Electronic Properties of a Molecule When It Is Wired into a Circuit. J. Phys. Chem. B 2002, 106 (34), 8609-8614.

(64) Wang, W.; Lee, T.; Reed, A. Mechanism of Electron Conduction in Self-Assembled Alkanethiol Monolayer Devices. Phys. Rev. B: Condens. Matter Mater. Phys. 2003, 68 (12), 1-7.

(65) Engelkes, V. B.; Beebe, J. M.; Frisbie, C. D. Length-Dependent Transport in Molecular Junctions Based on SAMs of Alkanethiols and Alkanedithiols: Effect of Metal Work Function and Applied Bias on Tunneling Efficiency and Contact Resistance. J. Am. Chem. Soc. 2004, 126 (43), 14287-14296.

(66) Salomon, A.; Boecking, T.; Chan, C. K.; Amy, F.; Girshevitz, O.; Cahen, D.; Kahn, A. How Do Electronic Carriers Cross Si-Bound Alkyl Monolayers? Phys. Rev. Lett. 2005, 95 (26), 1-4.

(67) Papp, E.; Jelenfi, D. P.; Veszeli, M. T.; Vattay, G. A Landauer Formula for Bioelectronic Applications. Biomolecules 2019, 9 (10), 599. 
(68) Ho Choi, S.; Kim, B.; Frisbie, C. D. Electrical Resistance of Long Conjugated Molecular Wires. Science (Washington, DC, U. S.) 2008, 320 (5882), 1482-1486.

(69) Yan, H.; Bergren, A. J.; McCreery, R.; Della Rocca, M. L.; Martin, P.; Lafarge, P.; Lacroix, J. C. Activationless Charge Transport across 4.5 to $22 \mathrm{Nm}$ in Molecular Electronic Junctions. Proc. Natl. Acad. Sci. U. S. A. 2013, 110 (14), 5326-5330.

(70) Nguyen, Q. V.; Martin, P.; Frath, D.; Della Rocca, M. L.; Lafolet, F.; Bellinck, S.; Lafarge, P.; Lacroix, J.-C. Highly Efficient Long-Range Electron Transport in a Viologen-Based Molecular Junction. J. Am. Chem. Soc. 2018, 140 (32), 10131-10134.

(71) Luo, L.; Choi, S. H.; Frisbie, C. D. Probing Hopping Conduction in Conjugated Molecular Wires Connected to Metal Electrodes $\dagger$. Chem. Mater. 2011, 23 (3), 631-645.

(72) Chen, X.; Salim, T.; Zhang, Z.; Yu, X.; Volkova, I.; Nijhuis, C. A. Large Increase in the Dielectric Constant and Partial Loss of Coherence Increases Tunneling Rates across Molecular Wires. ACS Appl. Mater. Interfaces 2020, 12 (40), 45111-45121. 